\title{
ON FACTORIZATIONS OF SMOOTH NONNEGATIVE MATRIX-VALUES FUNCTIONS AND ON SMOOTH FUNCTIONS WITH VALUES IN POLYHEDRA
}

\author{
N.V. KRYLOV
}

\begin{abstract}
We discuss the possibility to represent smooth nonnegative matrix-valued functions as finite linear combinations of fixed matrices with positive real-valued coefficients whose square roots are Lipschitz continuous. This issue is reduced to a similar problem for smooth functions with values in a polyhedron.
\end{abstract}

\section{Motivation}

One of the main goals of the article is to understand what kind of optimal control problems of diffusion processes is covered by the results of [3] and [7], where the processes are given by Itô equations in a "special" form, such that in the corresponding Bellman equation the second order part is represented as the sum of second-order derivatives with respect to fixed vectors (independent of the control parameter) times squares of real-valued functions that are Lipschitz continuous with respect to the space variables. Roughly speaking the answer is that all control problems with twice continuously differentiable diffusion matrices fall into the scheme of [3] and [7] whenever property (A) holds: these matrices for all values of control and time and space variables belong to a fixed polyhedron in the set of symmetric nonnegative matrices. In the author's opinion the control problems with property (A) are the only ones which admit finite-difference approximations with monotone schemes based on scaling of a fixed mesh.

For functions $w(z)$ given in a Euclidean space and vectors $\xi$ in that space set

$$
w_{(\xi)}=(\xi, \nabla w)=\sum_{i} \xi^{i} w_{z^{i}}, \quad w_{(\xi)(\xi)}=\sum_{i, j} \xi^{i} \xi^{j} w_{z^{i} z^{j}}
$$

In many situations one needs to represent a $d \times d$ nonnegative symmetric matrix $u$ as the square of a matrix or more generally as the product $v v^{*}$, where $v$ is not necessarily a square matrix. If $u=\left(u^{i j}\right)=v v^{*}$ and $v=\left(v^{i k}\right)$ and for each $k$ we introduce the vector $v^{k}=\left(v^{i k}\right) \in \mathbb{R}^{d}$, then for any smooth

1991 Mathematics Subject Classification. 15A99, 65M06.

Key words and phrases. Finite-difference approximations, polyhedra, diagonally dominant matrices.

The work was partially supported by NSF Grant DMS-0653121. 
$f(x)$ given on $\mathbb{R}^{d}$ and the operator

$$
L f:=\sum_{i, j} u^{i j} f_{x^{i} x^{j}}
$$

we have

$$
L f=\sum_{k} f_{\left(v^{k}\right)\left(v^{k}\right)} .
$$

In fact, as is easy to see having (1.2) for all smooth $f$ is equivalent to the validity of the formula $u=v v^{*}$.

There are very many $v$ such that $u=v v^{*}$ and then a few questions arise:

(i) if $u$ is a measurable function of a parameter, can one find a measurable $v ?$

(ii) if $u$ is smooth, can one find a Lipschitz continuous $v$ ?

The answer to the first question is easy and positive. Indeed, one can take

$$
v=c \int_{0}^{\infty} t^{-3 / 2}\left(e^{-u t}-1\right) d t
$$

where $c$ is an appropriate constant. This defines $v$ as the square root of $u$. Since long ago it is known that the square root of a twice differentiable nonnegative matrix-valued function is Lipschitz continuous (see [4, [10]). This result was used in the investigation of solvability of degenerate elliptic and parabolic second-order equations by using probabilistic or classical approaches.

However, there are applications in which formula (1.2) is not very convenient. One of these applications is related to finite-difference approximations of solutions to elliptic and parabolic equations with variable coefficients $u^{i j}$. Formula (1.2) suggests replacing $f_{\left(v^{k}\right)\left(v^{k}\right)}$ with the second-order difference along vectors $v^{k}$ and if $v^{k}$ vary, it may be impossible to find a reasonable mesh on which the approximation operator would make sense. This problem does not arise if $v^{k}=\sigma_{k} \gamma^{k}$, where $\gamma^{k}$ are constant vectors and $\sigma_{k}$ are real-valued functions, because then

$$
L f=\sum_{k} \sigma_{k}^{2} f_{\left(\gamma^{k}\right)\left(\gamma^{k}\right)}
$$

and one can concentrate on meshes that are obtained by contracting

$$
\left\{\sum_{k} n_{k} \gamma_{k}: n_{k}=0, \pm 1, \ldots\right\} \text {. }
$$

According to Remark 2.1 of [3] considering operators $L$ in form (1.3) is rather realistic from the point of view of numerical approximations. It turns out that if we fix a finite subset $B \subset \mathbb{R}^{d}$, such that $\operatorname{Span} B=\mathbb{R}^{d}$, and if $L$ from (1.1) admits a finite-difference approximation

$$
L_{h} f(0)=\sum_{y \in B} p_{h}(y) f(x+h y) \rightarrow L f(0) \quad \text { as } \quad h \downarrow 0, \quad \forall f \in C^{2}
$$


and $L_{h}$ are monotone, that is $L_{h} f(0) \geq 0$ whenever $f(x) \geq f(0)$ on $\mathbb{R}^{d}$, then automatically $L$ is written in the form (1.3) with some $\gamma^{k} \in B$.

Now the question is: If $u=u(x)$, under which assumptions can one find constant vectors $\gamma_{k}$ 's and functions $\sigma_{k}^{2}(x)$ in order for (1.3) to hold? Perhaps, Motzkin and Wasow (see [9]) were the first to address this question in the framework of finite-difference approximation. They proved (see also Lemma 17.13 of [5]) that if we denote by $S[\lambda, \Lambda]$ the (closed) set of positive $d \times d$ matrices with eigenvalues lying in the interval $[\lambda, \Lambda]$, where $0<\lambda \leq \Lambda$, then there exist a finite set of unit vectors $\gamma^{1}, \ldots, \gamma^{m} \in \mathbb{R}^{d_{2}}$ and numbers $0<\lambda^{*}<\Lambda^{*}$, such that any $u \in S[\lambda, \Lambda]$ can be written in the form

$$
u^{i j}=\sum_{k=1}^{m} \beta_{k} \gamma^{i k} \gamma^{j k},
$$

where the numbers $\beta_{k}$ satisfy the inequalities $\lambda^{*} \leq \beta_{k} \leq \Lambda^{*}$. In that case (1.3) holds with $\sigma_{k}^{2}=\beta_{k}$. This fact was used in the development of the theory of fully nonlinear second-order elliptic and parabolic equations.

One can give a quite easy explanation of this result. If we take any $\lambda_{1}<\lambda$ and $\Lambda_{1}>\Lambda$, the set $S\left[\lambda_{1}, \Lambda_{1}\right]$ will contain an open polyhedron $P^{o}$ containing $S[\lambda, \Lambda]$. Each point of a polyhedron is represented as a convex combination of its vertices and one easily obtains (1.4), for instance, as in the proof of Lemma 5.5 .4 of 6 .

With a little more effort one can get more convenient representations. We claim that given any open bounded polyhedron $P^{o}$ in a Euclidean space $E$ of points $y$ with vertices, say $y_{1}, \ldots ., y_{n}$, there exist infinitely differentiable functions $p_{k}(y)>0, k=1, \ldots, n$, such that for any $y \in P^{o}$

$$
y=\sum_{k} p_{k}(y) y_{k}, \quad \sum_{k} p_{k}(y)=1 .
$$

This fact is proved by induction on the dimension of $P^{o}$. First, without losing generality one may assume that the volume of $P^{o}$ in $E$ is strictly greater than zero. Then, assume that the fact is true for any face of $P^{o}$ and then solve Laplace's equation $\Delta p_{k}=0$ in $P^{o}$ with boundary condition $p_{k}(y)=\bar{p}_{k}(y)$ on $\partial P^{o}$, where $\bar{p}_{k}$ is the weight of the vertex $y_{k}$ in the representation of $y \in \partial P^{o}$, which is supposed to hold by the induction hypothesis. Of course, if $y \in \partial P^{o}$ and $y_{k}$ do not belong to the same face, we set $\bar{p}_{k}(y)=0$. Then by the well-known properties of harmonic functions $p_{k}>0$ in $P^{o}$, they are infinitely differentiable in $P^{o}$ and since

$$
\Delta \sum_{k} p_{k}(y) y_{k}=0, \quad \text { in } \quad P^{o} \quad \text { and } \quad \sum_{k} p_{k}(y) y_{k}=y \quad \text { on } \quad \partial P^{o}
$$

and $\Delta y=0$, by uniqueness we have the first relation in (1.5). The second one is obtained similarly from the fact that it holds on $\partial P^{o}$ and $\Delta 1=0$. 
After having proved the claim we return to the original $P^{o}$ and write for any $u \in P^{o}$

$$
u=\sum_{k} p_{k}(u) u_{k}, \quad \sum_{k} p_{k}(u)=1,
$$

where $u_{k} \in P^{o} \subset S\left[\lambda_{1}, \Lambda_{1}\right], p_{k}$ are infinitely differentiable in $P^{o}$, in particular, in $S[\lambda, \Lambda], p_{k}>0$ in $P^{o}$, in particular, they are bounded away from zero on the closed set $S[\lambda, \Lambda]$. Now to obtain (1.4) from (1.6) it only remains to recall that if $\xi_{k i}, i=1, \ldots, d$, are unit eigenvectors of $u_{k}$ with eigenvalues $\mu_{k i}$, then $\lambda_{1} \leq \mu_{k i} \leq \Lambda_{1}$ and

$$
u_{k}=\sum_{i} \mu_{k i} \xi_{k i} \xi_{k i}^{*}
$$

The above construction of $p_{k}(u)$ has a very substantial advantage over the original one in [9] (or [5] and [6]). Namely, it is seen that if $u=u(x)$ is a smooth function of a parameter $x$, then in the representation

$$
u(x)=\sum_{k} p_{k}(u(x)) u_{k}
$$

or in the implied representation (1.3) the functions $p_{k}(u(x)), p_{k}^{1 / 2}(u(x))$, and $\sigma_{k}(x)$ are as smooth as $u(x)$ is.

We see that from the point of view of the possibility of applying numerical approximations to uniformly nondegenerate equations the situation looks quite promising. For degenerate equations and fully nonlinear equations the situation is much more complex. In this case we again may try to prove (1.6) with $p_{k}$ such that $p_{k}^{1 / 2}(u)$ is Lipschitz continuous in $u$. However, this is impossible even if $d=1$ and $S[\lambda, \Lambda]=[0,1]$. In this case, naturally $u_{1}=0$, $u_{2}=1$, and $p_{2}(u)=u$, so that $p_{2}^{1 / 2}(u)$ is not Lipschitz continuous.

On the other hand, in numerical approximation or probabilistic approach one needs $p_{k}^{1 / 2}(u(x))$ to be Lipschitz continuous function of $x$, rather than $p_{k}^{1 / 2}(u)$ to be Lipschitz continuous function of $u$. This slight difference makes the problem solvable in some cases. For instance, in the above case that $d=1$ it is known that for any nonnegative twice continuously differentiable function $u(x)$ its square root $u^{1 / 2}(x)$ is Lipschitz continuous.

Another example is given by the functions with values in the set of the so-called diagonally dominant nonnegative symmetric matrices, which are quite popular in the literature (see [1], [8]). These are the ones with the property

$$
2 u^{i i} \geq \sum_{j=1}^{d}\left|u^{i j}\right|, \quad i=1, \ldots, d .
$$

Let $D$ be the set of symmetric matrices satisfying (1.8) and such that trace $u=1$. The author heard some doubts that, say the results of [3] are applicable to equations whose variable coefficients of second order derivatives form matrices of class $D$. The point is that the equations in [3] are 
assumed to have the structure associated with (1.3) with Lipschitz continuous $\sigma_{k}$. A naive way fails to take $e_{1}, \ldots, e_{d}$ as the standard basis vectors in $\mathbb{R}^{d}$ and write a representation like (1.4) for a $D$-valued function $u(x)$ as (see [1])

$$
\begin{gathered}
u(x)=\sum_{i \neq j}\left[\left(u^{i i}(x)-\left|u^{i j}(x)\right|\right) e_{i} e_{i}^{*}+(1 / 2)\left(u^{i j}(x)\right)^{+}\left(e_{i}+e_{j}\right)\left(e_{i}+e_{j}\right)^{*}\right. \\
\left.+(1 / 2)\left(u^{i j}(x)\right)^{-}\left(e_{i}-e_{j}\right)\left(e_{i}-e_{j}\right)^{*}\right],
\end{gathered}
$$

where we used the notation $a^{ \pm}=(1 / 2)(|a| \pm a)$. The reason for the failure is that no smoothness assumptions on $u(x)$ can guarantee that $\left[\left(u^{i j}\right)^{+}\right]^{1 / 2}$ is Lipschitz continuous for $i \neq j$. One needs a nontrivial structural assumption for that.

Nevertheless, in 7] for $d=2$ the author gave explicit formulas for representing twice continuously differentiable $D$-valued functions in the form (1.7) with Lipschitz continuous $p_{k}^{1 / 2}(u(x))$. The result of the present article shows that such representation exists for any $d$. In addition, it turns out that the set of diagonally dominant matrices can be replaced with any set which is a polyhedron in the set of $d \times d$ matrices. By the way, observe that (1.8) can be equivalently written as

$$
2 u^{i i} \geq \sum_{j=1}^{d} \varepsilon^{i j} u^{i j}, \quad i=1, \ldots, d, \varepsilon^{i j}= \pm 1 .
$$

In $D$ we also have trace $u=1$. Therefore, the bounded set $D$ is described by means of finitely many linear equalities and inequalities, and hence $D$ is a polyhedron in the space of $d \times d$ matrices. Speaking about the case that $d=2$, it is also worth noting that in [2] an efficient algorithm is introduced for approximating arbitrary $2 \times 2$ nonnegative matrices with matrices of the form $\sum_{k} p_{k} \xi_{k} \xi_{k}^{*}$, where $\xi_{k} \in \mathbb{R}^{2}$.

Finally, we reiterate that representation (1.7) leads to (1.3) and the latter means that we have the following factorization:

$$
u=v v^{*}, \quad \text { where } \quad v^{i k}=\sigma_{k} \gamma^{i k} .
$$

Starting from this point we forget about matrices and work with functions having values in a polyhedron. Our main results are presented in Section 2 , Theorem 2.1 is proved in Section 2 and Theorem 2.2 is proved in Section 4 , Section 3 contains an investigation of an auxiliary function some additional information on which is provided in Section 5 .

The author discussed the article with Hongjie Dong whose comments are greatly appreciated.

\section{MAin Results}

Let $P$ be a closed bounded convex polyhedron in $\mathbb{R}^{d}$ with distinct vertices $a_{1}, \ldots, a_{n}$, where $n \geq 2$. Let $d_{1} \geq 1$ be an integer. 
Theorem 2.1. Let $u(y)$ be a P-valued functions defined on $\mathbb{R}^{d_{1}}$. Assume that the first and second order derivatives of $u$ are bounded and continuous on $\mathbb{R}^{d_{1}}$. Then there exist real valued nonnegative functions $u_{1}(y), \ldots, u_{n}(y)$ such that

$$
\sum_{k} u_{k}(y) \equiv 1, \quad u(y) \equiv \sum_{k} u_{k}(y) a_{k},
$$

and $u_{k}^{1 / 2}$ are Lipschitz continuous on $\mathbb{R}^{d_{1}}$ with a constant which depends only on $P$ and $\sup \left\{\left|u_{(\eta)(\eta)}(y)\right|:|\eta|=1, y \in \mathbb{R}^{d_{1}}\right\}$.

Clearly the following assumption which we keep throughout the paper does not restrict generality:

$$
a_{1}=0, \quad \operatorname{Span}\left(a_{2}, \ldots, a_{n}\right)=\mathbb{R}^{d} .
$$

To prove Theorem 2.1 we need the following result. For $\xi \in \mathbb{R}^{d} \backslash\{0\}$ and $x \in P$ denote by $d(x, \xi)$ the distance from $x$ to $\partial P$ along the ray $x+t \xi /|\xi|$, $t \geq 0$. Introduce, $P^{o}$ as the interior of $P$.

Denote by $\Phi$ the set of $d$-1-dimensional faces of $P$ and for $\Gamma \in \Phi$ and $x \in P^{o}$ introduce $d_{\Gamma}(x)$ as the distance from $x$ to $\Gamma$. Also let $n_{\Gamma}$ be a unit normal vector to $\Gamma$.

Theorem 2.2. On $P$ there exist Lipschitz continuous nonnegative functions $p_{1}(x), \ldots, p_{n}(x)$ which are infinitely differentiable in $P^{o}$ and such that

(i) $p_{k}>0$ in $P^{o}$;

(ii) in $P$ we have

$$
\sum_{k} p_{k}(x)=1, \quad x=\sum_{k} p_{k}(x) a_{k}
$$

(iii) for any $\xi \in \mathbb{R}^{d}$ and $x \in P^{o}$ we have

$$
\frac{\left|p_{k(\xi)}(x)\right|}{p_{k}^{1 / 2}(x)} \leq N \max _{\Gamma \in \Phi} \frac{\left|\left(n_{\Gamma}, \xi\right)\right|}{d_{\Gamma}^{1 / 2}(x)},
$$

where $N$ is a finite constant depending only on $P$.

Proof of Theorem 2.1. Take a point $x_{0} \in P^{o}$ and for $t \in(0,1)$ set $u_{t}(y)=t u(y)+(1-t) x_{0}$. Then $u_{t}$ takes values in $P^{o}$. Assume that for $P^{o}$-valued functions the statement of Theorem 2.1 is true. Then, for each $t \in(0,1)$ there exist real valued nonnegative functions $u_{t 1}(y), \ldots, u_{t n}(y)$ such that

$$
\sum_{k} u_{t k}(y) \equiv 1, \quad u_{t}(y) \equiv \sum_{k} u_{t k}(y) a_{k}
$$

and $u_{t k}^{1 / 2}$ are Lipschitz continuous on $\mathbb{R}^{d_{1}}$ with a constant independent of $t$. By the Arzelà-Ascoli theorem it follows that there exists a sequence $t_{n} \uparrow 1$ such that $u_{t_{n} k}(y)$ converge to some functions $u_{k}(y)$ for each $y$ and $u_{k}^{1 / 2}$ are Lipschitz continuous. Obviously, these are the functions which we need. We see that without losing generality we may assume that $u(y) \in P^{o}$ for all $y$. 
We will be using the well-known fact that if we have a nonnegative twice continuously differentiable function $f(y)$ given on $\mathbb{R}^{d_{1}}$ and having bounded second-order derivatives, then for any $y \in \mathbb{R}^{d_{1}}$

$$
|\nabla f(y)|^{2} \leq 4 f(y) \sup \left\{\left|f_{(\eta)(\eta)}(z):\right| \eta \mid=1, z \in \mathbb{R}^{d_{1}}\right\} .
$$

Now take $p_{k}$ from Theorem 2.2 and set $u_{k}(y)=p_{k}(u(y))$. Then the equations (2.1) obviously hold. Since $p_{k}$ are positive and infinitely differentiable in $P^{o}, u_{k}$ are positive and continuously differentiable in $\mathbb{R}^{d_{1}}$. Therefore, to estimate the Lipschitz constant of $u_{k}^{1 / 2}$ it suffices to estimate its first order directional derivatives.

Fix a $y, \eta \in \mathbb{R}^{d_{1}}$ with $|\eta|=1$ and set $x=u(y), \xi=u_{(\eta)}(y)$. Then by (2.2)

$$
2\left|\left(u_{k}^{1 / 2}\right)_{(\eta)}(y)\right|=\frac{\left|p_{k(\xi)}(x)\right|}{p_{k}^{1 / 2}(x)} \leq N \max _{\Gamma \in \Phi} \frac{\left|\left(n_{\Gamma}, \xi\right)\right|}{d_{\Gamma}^{1 / 2}(x)} .
$$

Next, take a face $\Gamma \in \Phi$ and let it be given as $\left\{x:\left(n_{\Gamma}, x\right)=b\right\}$, where $b$ is a constant. By multiplying $n_{\Gamma}$ and $b$ by -1 if needed we may assume that

$$
\left(n_{\Gamma}, w\right) \geq b \quad \forall w \in P .
$$

Then $f(z):=\left(n_{\Gamma}, u(z)\right)-b$ is a nonnegative twice continuously differentiable function on $\mathbb{R}^{d_{1}}$. By the above

$$
\left|\left(n_{\Gamma}, \xi\right)\right|^{2}=\left|f_{(\eta)}(y)\right|^{2} \leq N f(y)=N\left|\left(n_{\Gamma}, x\right)-b\right|=N d_{\Gamma}(x),
$$

where

$$
N=4 \sup _{|\eta|=1, z}\left|u_{(\eta)(\eta)}(z)\right| .
$$

This and (2.3) bring the proof of the theorem to an end.

\section{An AUXILIARY FUnCTION}

For $x \in P^{o}$ define

$$
U(x)=\max \left\{\sum_{i=1}^{n} \ln p_{i}: p_{i}>0, \sum_{i=1}^{n} p_{i}=1, \quad \sum_{i=1}^{n} p_{i} a_{i}=x\right\} .
$$

Obviously, $U \leq 0$ and for each $x \in P^{o}$ there exists $p_{1}, \ldots, p_{n}$ achieving the maximum.

Lemma 3.1. (i) The function $U$ is strictly concave and therefore continuous in $P^{o}$.

(ii) For each $x \in P^{o}$ there exists a unique set $p_{1}(x), \ldots, p_{n}(x)>0$ such that

$$
\sum_{i=1}^{n} p_{i}(x)=1, \quad \sum_{i=1}^{n} p_{i}(x) a_{i}=x, \quad U(x)=\sum_{i} \ln p_{i}(x) .
$$

(iii) The functions $p_{1}(x), \ldots, p_{n}(x)$ are continuous in $P^{o}$. 
Proof. (i) Take $x, y \in P^{o}, t, s \in(0,1)$, such that $t+s=1$, and let $p_{1}, \ldots, p_{n}$ and $q_{1}, \ldots, q_{n}$ be some sets achieving the maximums for $x$ and $y$ respectively. Then for $r_{i}=t p_{i}+s q_{i}$ we have

$$
\sum_{i} r_{i}=1, \quad \sum_{i} r_{i} a_{i}=t x+s y .
$$

Hence,

$$
\begin{gathered}
U(t x+s y) \geq \sum_{i} \ln \left(t p_{i}+s q_{i}\right) \geq t \sum_{i} \ln p_{i}+s \sum_{i} \ln q_{i} \\
=t U(x)+s U(y),
\end{gathered}
$$

where the second inequality is strict if $p_{i} \neq q_{i}$ for at least one $i$. This is certainly the case if $x \neq y$, which proves (i). Another case would appear if $x=y$ and we assumed that there are two different sets $p_{1}, \ldots, p_{n}$ and $q_{1}, \ldots, q_{n}$ achieving $U(x)$. But then the above computations would lead to a wrong conclusion that $U(x)>U(x)$. This proves (ii).

Finally (iii) follows from the continuity of $U(x)$ and assertion (ii). The lemma is proved.

Lemma 3.2. The function $U$ is continuously differentiable in $P^{o}$ and for any $x \in P^{o}$ and $\xi \in \mathbb{R}^{d}$, which is represented as

$$
\xi=\sum_{k} q_{k}\left(x-a_{k}\right)
$$

with some numbers $q_{k}$, we have

$$
\sum_{k} \frac{q_{k}}{p_{k}(x)}=n \sum_{k} q_{k}-U_{(\xi)}(x) .
$$

In particular, as $\xi=x-a_{k}$,

$$
\frac{1}{p_{k}(x)}=n-U_{\left(x-a_{k}\right)}(x) .
$$

Proof. Fix an $x_{0} \in P^{o}$ and let $\lambda \in \mathbb{R}^{d}$ be such that the graph of the function $\left(\lambda, x-x_{0}\right)+U\left(x_{0}\right)$ is a supporting plane for the graph of $U(x)$ at $\left(x_{0}, U\left(x_{0}\right)\right)$. Set $b:=\sum_{k} q_{k}$ and write

$$
x_{0}+t \xi=\sum_{k}\left[(1+b t) p_{k}\left(x_{0}\right)-t q_{k}\right] a_{k} .
$$

For sufficiently small $t$ we have $(1+b t) p_{k}\left(x_{0}\right)-t q_{k}>0$ and

$$
\sum_{k}\left[(1+b t) p_{k}\left(x_{0}\right)-t q_{k}\right]=1 \text {. }
$$

It follows that for small $t$

$$
t(\lambda, \xi)+U\left(x_{0}\right) \geq U\left(x_{0}+t \xi\right) \geq \sum_{k} \ln \left[(1+b t) p_{k}\left(x_{0}\right)-t q_{k}\right]
$$


with equalities instead of the inequalities for $t=0$. By differentiating at $t=0$ the extreme terms we find

$$
(\lambda, \xi)=n \sum_{k} q_{k}-\sum_{k} \frac{q_{k}}{p_{k}\left(x_{0}\right)} .
$$

If there is another vector $\mu \in \mathbb{R}^{d}$ such that the graph of the function $(\mu, x-$ $\left.x_{0}\right)+U\left(x_{0}\right)$ is a supporting plane for the graph of $U(x)$ at $\left(x_{0}, U\left(x_{0}\right)\right)$, then the above formula implies that $\lambda-\mu \perp \xi$. This holds for any $\xi$ admitting representation (3.1) with $x_{0}$ in place of $x$. Since

$$
\operatorname{Span}\left\{\left(x_{0}-a_{1}\right)-\left(x_{0}-a_{2}\right), \ldots,\left(x_{0}-a_{1}\right)-\left(x_{0}-a_{n}\right)\right\}=\mathbb{R}^{d},
$$

any $\xi$ has the said property, and hence $\lambda=\mu$.

Thus, for each point $x_{0} \in P^{o}$ there is only one supporting plane at $\left(x_{0}, U\left(x_{0}\right)\right)$. This and the concavity of $U$ implies that $U$ is continuously differentiable, $\lambda=\nabla U\left(x_{0}\right)$, and the lemma is proved.

Corollary 3.3. Take any representation

$$
x=\sum_{k} q_{k} a_{k} \quad \text { with } \quad \sum_{k} q_{k}=1 .
$$

Then

$$
\sum_{k} \frac{q_{k}}{p_{k}(x)}=n
$$

Indeed, it suffices to observe that $\xi=0$ in (3.2).

Lemma 3.4. The functions $U, p_{k}$ are infinitely differentiable in $P^{o}$.

Proof. Denote $\lambda(x)=\nabla U(x)$. Then

$$
p_{k}(x)=\frac{1}{n-\left(x-a_{k}, \lambda(x)\right)},
$$

and $\lambda(x)$ satisfies

$$
F(\lambda(x), x)=0
$$

where

$$
F(\lambda, x)=\left(F^{i}(\lambda, x), i=1, \ldots, d\right), \quad F^{i}(\lambda, x)=\sum_{k} \frac{1}{n-\left(x-a_{k}, \lambda\right)}\left(x^{i}-a_{k}^{i}\right) .
$$

We have

$$
\frac{\partial}{\partial \lambda^{j}} F^{i}(\lambda, x)=\sum_{k} \frac{1}{\left(n-\left(x-a_{k}, \lambda\right)\right)^{2}}\left(x^{j}-a_{k}^{j}\right)\left(x^{i}-a_{k}^{i}\right) .
$$

By (3.4) there is no nonzero vectors $\eta$ that are orthogonal to all $x-a_{k}$. It follows that the matrix with the entries $\frac{\partial}{\partial \lambda^{j}} F^{i}(\lambda, x)$ is nondegenerate, $\lambda(x)$ is infinitely differentiable by the implicit function theorem and the lemma is proved. 
Lemma 3.5. Let $x \in P^{o}, \xi \in \mathbb{R}^{d}$. Then

$$
U_{(\xi)(\xi)}(x)=-\sum_{k} \frac{\left(p_{k(\xi)}(x)\right)^{2}}{p_{k}^{2}(x)} .
$$

Furthermore, if $\xi=\sum_{k} q_{k}\left(a_{k}-x\right)$ for some numbers $q_{k}$, then

$$
\begin{gathered}
-U_{(\xi)(\xi)}(x)=\sum_{k} \frac{p_{k(\xi)}(x) q_{k}}{p_{k}^{2}(x)}-U_{(\xi)}(x) \sum_{k} q_{k}, \\
\sum_{k} \frac{\left(p_{k(\xi)}(x)\right)^{2}}{p_{k}^{2}(x)} \leq(n+1) \sum_{k} \frac{q_{k}^{2}}{p_{k}^{2}(x)} .
\end{gathered}
$$

Finally, $\left|p_{k\left(a_{k}-x\right)}(x)\right| \leq(n+1)^{1 / 2}$ for any $k=1, \ldots, n$.

Proof. By differentiating (3.3) we find

$$
\frac{p_{k(\xi)}(x)}{p_{k}^{2}(x)}=-U_{\left(a_{k}-x\right)(\xi)}(x)+U_{(\xi)}(x) .
$$

By multiplying this equality by $q_{k}$ and summing up with respect to $k$ we get (3.6) provided that $\xi=\sum_{k} q_{k}\left(a_{k}-x\right)$. Differentiating $\sum_{k} p_{k}(x) a_{k}=x$ and $\sum_{k} p_{k}(x)=1$ yields

$$
\sum_{k} p_{k(\xi)}(x) a_{k}=\xi, \quad \sum_{k} p_{k(\xi)}(x)=0, \quad \xi=\sum_{k} p_{k(\xi)}(x)\left(a_{k}-x\right),
$$

which allows us to use (3.6) with $q_{k}=p_{k(\xi)}$ and obtain (3.5).

Next, the right-hand side of (3.6) equals

$$
\sum_{k} \frac{q_{k}}{p_{k}}\left(\frac{p_{k(\xi)}}{p_{k}}-p_{k} U_{(\xi)}\right) \text {. }
$$

Its square by Hölder's inequality is less than

$$
\sum_{k} \frac{q_{k}^{2}}{p_{k}^{2}} \sum_{k}\left(\frac{\left(p_{k(\xi)}\right)^{2}}{p_{k}^{2}}-2 p_{k(\xi)} U_{(\xi)}+p_{k}^{2} U_{(\xi)}^{2}\right) .
$$

We recall (3.5) and observe that

$$
\sum_{k} p_{k(\xi)}=0, \quad \sum_{k} p_{k}^{2} \leq 1, \quad U_{(\xi)}^{2}=\left(\sum_{k} \frac{p_{k(\xi)}}{p_{k}}\right)^{2} \leq-n U_{(\xi)(\xi)} .
$$

Then we find that

$$
U_{(\xi)(\xi)}^{2} \leq(n+1)\left|U_{(\xi)(\xi)}\right| \sum_{k} \frac{q_{k}^{2}}{p_{k}^{2}}
$$

which is equivalent to (3.7).

The last assertion of the lemma is obtained by taking $\xi=a_{k}-x$ in (3.7). The lemma is proved. 
Theorem 3.6. For $\xi \neq 0$ in $P^{o}$ we have

$$
U_{(\xi)(\xi)}(x) \geq-\left(n+4 n^{2}\right) \frac{|\xi|^{2}}{d^{2}(x, \xi) \wedge d^{2}(x,-\xi)} .
$$

In particular, for any $x$ in $P^{o}$ we have

$$
\frac{\left|p_{k(\xi)}(x)\right|}{p_{k}(x)} \leq N \frac{|\xi|}{d(x, \xi) \wedge d(x,-\xi)}
$$

where $N=\left(n+4 n^{2}\right)^{1 / 2}$.

Proof. Without losing generality we assume that $|\xi|=1$, take $x \in P^{o}$, and set

$$
y=x+d(x, \xi) \xi, \quad y(t)=(1-t) x+t y, \quad t \in(-\varepsilon, 1),
$$

where $\varepsilon>0$ is to be chosen later. Certainly there is a representation

$$
y=\sum_{k} q_{k} a_{k}, \quad q_{k} \geq 0, \quad \sum_{k} q_{k}=1 .
$$

Therefore, for sufficiently small $\varepsilon$ and all $t \in(-\varepsilon, 1)$ we have

$$
\begin{gathered}
y(t)=\sum_{k} a_{k}\left((1-t) p_{k}(x)+t q_{k}\right), \quad(1-t) p_{k}(x)+t q_{k}>0, \\
\sum_{k}\left((1-t) p_{k}(x)+t q_{k}\right)=1 .
\end{gathered}
$$

By definition,

$$
U(y(t)) \geq \sum_{k} \ln \left((1-t) p_{k}(x)+t q_{k}\right)
$$

with equality for $t=0$. Therefore, the second derivatives in $t$ at $t=0$ of the extreme terms are linked by a similar inequality, that is

$$
d^{2}(x, \xi) U_{(\xi)(\xi)}(x) \geq-\sum_{k} \frac{\left(p_{k}(x)-q_{k}\right)^{2}}{p_{k}^{2}(x)} \geq-n-\sum_{k} \frac{q_{k}^{2}}{p_{k}^{2}(x)} .
$$

In like manner for $z=x-d(x,-\xi) \xi$ we find

$$
\begin{gathered}
z=\sum_{k} r_{k} a_{k}, \quad r_{k} \geq 0, \quad \sum_{k} r_{k}=1, \\
d^{2}(x,-\xi) U_{(\xi)(\xi)}(x) \geq-n-\sum_{k} \frac{r_{k}^{2}}{p_{k}^{2}(x)} .
\end{gathered}
$$

However, for some $\alpha, \beta>0$ such that $\alpha y+\beta z=x$ and $\alpha+\beta=1$ we have

$$
x=\sum_{k}\left(\alpha q_{k}+\beta r_{k}\right) a_{k}, \quad \sum_{k}\left(\alpha q_{k}+\beta r_{k}\right)=1 .
$$

By Corollary 3.3

$$
\sum_{k} \frac{\alpha q_{k}+\beta r_{k}}{p_{k}(x)}=n
$$


It follows that

$$
\sum_{k} \frac{q_{k}^{2}}{p_{k}^{2}(x)} \leq\left(\sum_{k} \frac{q_{k}}{p_{k}(x)}\right)^{2} \leq \alpha^{-2} n^{2}, \quad \sum_{k} \frac{r_{k}^{2}}{p_{k}^{2}(x)} \leq \beta^{-2} n^{2}
$$

and hence at least one of

$$
\sum_{k} \frac{q_{k}^{2}}{p_{k}^{2}(x)}, \quad \sum_{k} \frac{r_{k}^{2}}{p_{k}^{2}(x)}
$$

is less than $4 n^{2}$. This yields the result and the theorem is proved.

Now we are going to get prepared to estimating the Lipschitz constants of $p_{k}$ 's. Recall that $a_{1}=0$ and let $P_{1}$ be the polyhedron with vertices $a_{2}, \ldots, a_{n}$, let $U_{n-1}(x)$ be the function $U$ defined relative to $P_{1}$, and let $P_{1}^{o}$ be the relative interior of $P_{1}$.

Lemma 3.7. Let $x \in P^{o}$ and let $\lambda>1$ be such that $\lambda x \in P_{1}^{o}$. Then

(i)

$$
U_{n-1}(\lambda x)-n \ln \lambda+\ln (\lambda-1) \leq U(x)
$$

(ii) we have an equality in (3.8) instead of the inequality if we take $\lambda=$ $\lambda(x):=\left(1-p_{1}(x)\right)^{-1}$.

Proof. Let $\bar{p}_{2}, \ldots, \bar{p}_{n}$ be the set that achieves $U_{n-1}(\lambda x)$. Then

$$
\sum_{i=2}^{n} \bar{p}_{i}=1, \quad \sum_{i=2}^{n} \bar{p}_{i} a_{i}=\lambda x
$$

Therefore, for $p_{i}:=\lambda^{-1} \bar{p}_{i}, i=2, \ldots, n$, and $p_{1}:=1-p_{2}-\ldots-p_{n}=1-\lambda^{-1}$ we have $p_{1}>0$, since $\lambda>1$, and

$$
\sum_{i=1}^{n} p_{i}=1, \quad \sum_{i=1}^{n} p_{i} a_{i}=\sum_{i=2}^{n} \lambda^{-1} \bar{p}_{i} a_{i}=x .
$$

By adding that

$$
U_{n-1}(\lambda x)=\sum_{i=2}^{n} \ln \bar{p}_{i}=(n-1) \ln \lambda+\sum_{i=1}^{n} \ln p_{i}-\ln \left(1-\lambda^{-1}\right) .
$$

we certainly obtain (3.8).

To prove assertion (ii) observe that for $\lambda=\lambda(x), p_{i}:=p_{i}(x)$, and $\bar{p}_{i}:=$ $\lambda p_{i}(x)$ we have (3.9) and $\bar{p}_{i}>0$. It follows that $\lambda x \in P_{1}^{o}$ and the first equality sign in (3.10) should be replaced with $\geq$. By combining this with (3.8) we get what we need. The lemma is proved.

Corollary 3.8. For $x \in P^{o}$ we have $\lambda(x) x \in P_{1}^{o}$ and the set $\lambda(x) p_{2}(x), \ldots$, $\lambda(x) p_{n}(x)$ achieves $U_{n-1}(\lambda(x) x)$, so that if for $y \in P_{1}^{o}$ we denote by $\bar{p}_{2}(y), \ldots$, $\bar{p}_{n}(y)$ the set that achieves $U_{n-1}(y)$, then for $x \in P^{o}$ and $k \geq 2$ we have

$$
p_{k}(x)=\left(1-p_{1}(x)\right) \bar{p}_{k}\left(\frac{x}{1-p_{1}(x)}\right) .
$$


Theorem 3.9. The functions $p_{1}(x), \ldots, p_{n}(x)$ are Lipschitz continuous in $P^{o}$ and, therefore, admit extensions to Lipschitz continuous functions in $P$.

Proof. We will be using the induction on $n$. If $n=2$ and say $P=[0,1]$, $a_{1}=0, a_{2}=1$, then $p_{1}(x)=1-x, p_{2}(x)=x$, and our assertion is true indeed.

Assume that our assertion is proved for all polyhedra with $n-1$ vertices. Then the functions $\bar{p}_{k}$ introduced in Corollary 3.8 are Lipschitz continuous in $P_{1}^{o}$. Since $\left|p_{1(x)}\right| \leq(n+1)^{1 / 2}$, from (3.11) we have that for $k \geq 2$ and $x \in P^{o}$

$$
\begin{gathered}
\left|p_{k(x)}(x)\right| \leq N+\left(1-p_{1}(x)\right) \lim _{\varepsilon \downarrow 0} \varepsilon^{-1}\left|\bar{p}_{k}\left(\frac{x+\varepsilon x}{1-p_{1}(x+\varepsilon x)}\right)-\bar{p}_{k}\left(\frac{x}{1-p_{1}(x)}\right)\right| \\
\leq N+N\left(1-p_{1}(x)\right) \lim _{\varepsilon \downarrow 0} \varepsilon^{-1}\left|\frac{x+\varepsilon x}{1-p_{1}(x+\varepsilon x)}-\frac{x}{1-p_{1}(x)}\right| \\
=N+N|x|\left|1+\frac{p_{1(x)}(x)}{1-p_{1}(x)}\right| \leq N+N \frac{|x|}{1-p_{1}(x)} .
\end{gathered}
$$

Thus, for any $\varepsilon>0, p_{k(x)}(x)$ are bounded as long as $|x| \geq \varepsilon$ and $k \geq 2$. Above we also used that $p_{1(x)}(x)$ is bounded.

Generally, $p_{k\left(x-a_{j}\right)}(x)$ are bounded as long as $\left|x-a_{j}\right| \geq \varepsilon$. In particular, $p_{1\left(x-a_{j}\right)}(x)$ are bounded for $\left|x-a_{j}\right| \geq \varepsilon$. We now claim that there exists an $\varepsilon>0$ and $N_{0}$ such that, for any unit $\xi \in \mathbb{R}^{d}$ and $x \in P$ one can find numbers $\eta_{1}, \ldots, \eta_{k}$ such that

$$
\xi=\sum_{k:\left|x-a_{k}\right| \geq \varepsilon} \eta_{k}\left(x-a_{k}\right), \quad\left|\eta_{k}\right| \leq N_{0}
$$

Indeed, if $\varepsilon$ is small enough the restriction of summation may exclude only one term with $k$ such that $\left|x-a_{k}\right|<\varepsilon$. Still the remaining set $\left\{x-a_{j}, j \neq k\right\}$ would be close to $\left\{a_{k}-a_{j}, j \neq k\right\}$ a subset of which forms a basis in $\mathbb{R}^{d}$. On the other hand, if there is nothing to exclude, our claim follows from (3.4).

This proves that $\left|p_{1(\xi)}(x)\right|$ is bounded for $x \in P^{o},|\xi|=1$. Of course, the same holds for other $\left|p_{k(\xi)}(x)\right|$ and the theorem is proved.

\section{Proof of Theorem 2.2}

First we introduce a few new objects. Let an integer $r \in[2, d]$ and let $\Gamma_{1}, \ldots, \Gamma_{r} \in \Phi$ be such that $n_{\Gamma_{1}}, \ldots, n_{\Gamma_{r}}$ are linearly independent. Then

$$
\left|n_{\Gamma_{r}}-\Pi_{\operatorname{Span}\left(n_{\Gamma_{1}}, \ldots, n_{\Gamma_{r-1}}\right)} n_{\Gamma_{r}}\right|>0,
$$

where $\Pi_{\mathcal{L}}$ is the orthogonal projection operator on a subspace $\mathcal{L} \in \mathbb{R}^{d}$. Since there are only finitely many such $r$ and $\Gamma_{1}, \ldots, \Gamma_{r} \in \Phi$, we see that there is a constant $\kappa \geq 1$ such that we always have

$$
\left|n_{\Gamma_{r}}-\Pi_{\operatorname{Span}\left(n_{\Gamma_{1}}, \ldots, n_{\Gamma_{r-1}}\right)} n_{\Gamma_{r}}\right| \geq \kappa^{-1} .
$$


For a $\zeta_{1}>0$ define recursively

$$
\zeta_{r}=2 \kappa \sum_{i=1}^{r-1} \zeta_{i} \quad r \geq 2
$$

Obviously, $\zeta_{r} \geq \zeta_{r-1}$ and $\zeta_{r}$ is a linear function of $\zeta_{1}$ so that we can choose and fix a $\zeta_{1}>0$ such that

$$
\gamma_{r}:=2^{r} \zeta_{r} \leq 1 / 2, \quad r=1, \ldots, d+1 .
$$

Set

$$
\varepsilon_{r}=\kappa^{-1} \gamma_{r} \quad\left(\leq \gamma_{r}\right) .
$$

Now fix $x$ and drop it in some notation. Take a $\Gamma \in \Phi$ for which $d(x, \xi) \wedge$ $d(x,-\xi)$ equals the distance from $x$ to $\Gamma$ along the line $x+t \xi|\xi|^{-1}, t \in \mathbb{R}$. Then

$$
d(x, \xi) \wedge d(x,-\xi)=|\xi| \frac{d_{\Gamma}(x)}{\left|\left(n_{\Gamma}, \xi\right)\right|} .
$$

Denote $\delta=\left|\nabla p_{k}\right|^{-1}$. There are two cases.

Case 1

Case 2

$$
\left|\left(n_{\Gamma}, \xi\right)\right| \geq \varepsilon_{1} \delta\left|p_{k(\xi)}\right| .
$$

$$
\left|\left(n_{\Gamma}, \xi\right)\right|<\varepsilon_{1} \delta\left|p_{k(\xi)}\right| .
$$

In the first case additionally assume that $p_{k}(x) \leq d_{\Gamma}(x)$. Then by Theorem 3.6

$$
\frac{\left|p_{k(\xi)}\right|}{p_{k}^{1 / 2}} \leq N|\xi| \frac{p_{k}^{1 / 2}}{d(x, \xi) \wedge d(x,-\xi)}=N \frac{p_{k}^{1 / 2}\left|\left(n_{\Gamma}, \xi\right)\right|}{d_{\Gamma}} \leq N \frac{\left|\left(n_{\Gamma}, \xi\right)\right|}{d_{\Gamma}^{1 / 2}} .
$$

On the other hand, if $p_{k}(x) \geq d_{\Gamma}(x)$, then

$$
\frac{\left|p_{k(\xi)}\right|}{p_{k}^{1 / 2}} \leq\left(\varepsilon_{1} \delta\right)^{-1} \frac{\left|\left(n_{\Gamma}, \xi\right)\right|}{p_{k}^{1 / 2}} \leq\left(\varepsilon_{1} \delta\right)^{-1} \frac{\left|\left(n_{\Gamma}, \xi\right)\right|}{d_{\Gamma}^{1 / 2}} .
$$

Thus, in Case 1 we have

$$
\frac{\left|p_{k(\xi)}\right|}{p_{k}^{1 / 2}} \leq N\left(\varepsilon_{1} \delta\right)^{-1} \frac{\left|\left(n_{\Gamma}, \xi\right)\right|}{d_{\Gamma}^{1 / 2}}
$$

which proves (2.2) since $\delta^{-1}$ is a bounded function.

In the rest of the proof we concentrate on Case 2. We will be using a recursive procedure. Denote $\xi_{1}=\xi, \Gamma_{1}=\Gamma, n_{1}=n_{\Gamma_{1}}$, and introduce

$$
\xi_{2}=\xi_{1}-n_{1}\left(n_{1}, \xi_{1}\right) \text {. }
$$

Observe that (Case 2)

$$
\begin{gathered}
\left|p_{k\left(\xi_{2}\right)}-p_{k\left(\xi_{1}\right)}\right|=\left|p_{k\left(n_{1}\right)}\right| \cdot\left|\left(n_{1}, \xi_{1}\right)\right| \leq \delta^{-1}\left|\left(n_{1}, \xi_{1}\right)\right|<\varepsilon_{1}\left|p_{k\left(\xi_{1}\right)}\right| \leq \gamma_{1}\left|p_{k\left(\xi_{1}\right)}\right|, \\
\left(1-\gamma_{1}\right)\left|p_{k\left(\xi_{1}\right)}\right|<\left|p_{k\left(\xi_{2}\right)}\right|<\left(1+\gamma_{1}\right)\left|p_{k\left(\xi_{1}\right)}\right| .
\end{gathered}
$$

In particular, $p_{k\left(\xi_{2}\right)} \neq 0$ and $\xi_{2} \neq 0$. Also in Case 2 we have $\xi_{2} \perp n_{1}$ and

$$
\left|\xi_{2}-\xi_{1}\right|=\left|\left(n_{1}, \xi_{1}\right)\right| \leq \varepsilon_{1} \delta\left|p_{k\left(\xi_{1}\right)}\right| \leq \gamma_{1} \delta\left|p_{k\left(\xi_{1}\right)}\right| .
$$


It follows that for an integer $r \geq 2$ we have $\Gamma_{i} \in \Phi, i=1, \ldots, r-1$, and vectors $\xi_{i} \neq 0, i=1, \ldots, r$, such that for $n_{i}$ being the normal vectors to $\Gamma_{i}$ we have

(i) $n_{1}, \ldots, n_{r-1}$ are linearly independent;

(ii) for $1 \leq j<i \leq r$ we have $\xi_{i} \perp n_{j}$;

(iii) for $2 \leq i \leq r$ we have

$$
\left(1-\gamma_{i-1}\right)\left|p_{k\left(\xi_{i-1}\right)}\right|<\left|p_{k\left(\xi_{i}\right)}\right|<\left(1+\gamma_{i-1}\right)\left|p_{k\left(\xi_{i-1}\right)}\right| ;
$$

(iv) for $2 \leq i \leq r$ we have $\left|\xi_{i}-\xi_{i-1}\right| \leq \gamma_{i-1} \delta\left|p_{k\left(\xi_{i-1}\right)}\right|$;

(v) for $1 \leq i \leq r-1$ we have $\left|\left(n_{i}, \xi_{i}\right)\right| \leq \varepsilon_{i} \delta\left|p_{k\left(\xi_{i}\right)}\right|$ :

(vi) for $1 \leq i \leq r-1$ the face $\Gamma_{i} \in \Phi$ is the one for which $d\left(x, \xi_{i}\right) \wedge d\left(x,-\xi_{i}\right)$ equals the distance from $x$ to $\Gamma_{i}$ along the line $x+t \xi_{i}\left|\xi_{i}\right|^{-1}, t \in \mathbb{R}$.

In light of (i) we have $r-1 \leq d$. Also observe that by virtue of (iii) and (iv) for $2 \leq i \leq r$ (recall that $\gamma_{j} \leq 1 / 2$ )

$$
\begin{gathered}
\left|p_{k\left(\xi_{i-1}\right)}\right| \leq\left|p_{k\left(\xi_{r}\right)}\right| \prod_{j=i-1}^{r-1}\left(1-\gamma_{j}\right)^{-1} \leq 2^{r-i+1}\left|p_{k\left(\xi_{r}\right)}\right|, \\
\left|\xi_{r}-\xi_{1}\right| \leq \sum_{i=2}^{r}\left|\xi_{i}-\xi_{i-1}\right| \leq \delta\left|p_{k\left(\xi_{r}\right)}\right| \sum_{i=2}^{r} \gamma_{i-1} 2^{r-i+1}=\varepsilon_{r} \delta\left|p_{k\left(\xi_{r}\right)}\right| / 2 .
\end{gathered}
$$

Now introduce $\Gamma_{r}$ as the face of $P$ for which $d\left(x, \xi_{r}\right) \wedge d\left(x,-\xi_{r}\right)$ equals the distance from $x$ to $\Gamma_{r}$ along the line $x+t \xi_{r}\left|\xi_{r}\right|^{-1}, t \in \mathbb{R}$. Set $n_{r}=n_{\Gamma_{r}}$ and first suppose that

Then as in Case 1

$$
\left|\left(n_{r}, \xi_{r}\right)\right| \geq \varepsilon_{r} \delta\left|p_{k\left(\xi_{r}\right)}\right|
$$

$$
\frac{\left|p_{k\left(\xi_{r}\right)}\right|}{p_{k}^{1 / 2}} \leq N\left(\varepsilon_{r} \delta\right)^{-1} \frac{\left|\left(n_{r}, \xi_{r}\right)\right|}{d_{\Gamma_{r}}^{1 / 2}} .
$$

Here by (4.1) the left-hand side dominates

$$
2^{-r} \frac{\left|p_{k\left(\xi_{1}\right)}\right|}{p_{k}^{1 / 2}}
$$

To estimate the right-hand side of (4.4) use (4.2) and (4.3) to get

$$
\left|\left(n_{r}, \xi_{r}\right)-\left(n_{r}, \xi_{1}\right)\right| \leq\left|\xi_{r}-\xi_{1}\right| \leq(1 / 2)\left|\left(n_{r}, \xi_{r}\right)\right| .
$$

Hence,

$$
\left|\left(n_{r}, \xi_{r}\right)\right| \leq 2\left|\left(n_{r}, \xi_{1}\right)\right|
$$

and going back to (4.4) we obtain

$$
\frac{\left|p_{k\left(\xi_{1}\right)}\right|}{p_{k}^{1 / 2}} \leq N \frac{\left|\left(n_{r}, \xi_{1}\right)\right|}{d_{\Gamma_{r}}^{1 / 2}},
$$

which proves (2.2).

In the situation that (4.3) is violated introduce

$$
\xi_{r+1}=\xi_{r}-f_{r}\left(n_{r}, \xi_{r}\right),
$$


where $f_{r}=h_{r} /\left|h_{r}\right|^{2}$ and

$$
h_{r}=n_{r}-\Pi_{\text {Span }\left(n_{1}, \ldots, n_{r-1}\right)} n_{r} .
$$

Observe that $h_{r} \neq 0$. Otherwise, $n_{r}$ would lie in $\operatorname{Span}\left(n_{1}, \ldots, n_{r-1}\right), \xi_{r}$ would be orthogonal also to $n_{r}$ and the line $x+t \xi_{r}\left|\xi_{r}\right|^{-1}, t \in \mathbb{R}$, would have never met $\Gamma_{r}$. In particular, property (i) holds with $r+1$ in place of $r$. By the definition of $\kappa$ we have $\left|h_{r}\right| \geq \kappa^{-1}$. Then $\left|f_{r}\right| \leq \kappa$ and since $\left|\left(n_{r}, \xi_{r}\right)\right|<\varepsilon_{r} \delta\left|p_{k\left(\xi_{r}\right)}\right|$, we have

$$
\left|p_{k\left(\xi_{r+1}\right)}-p_{k\left(\xi_{r}\right)}\right|=\left|p_{k\left(f_{r}\right)}\right| \cdot\left|\left(n_{r}, \xi_{r}\right)\right|<\kappa \varepsilon_{r}\left|p_{k\left(\xi_{r}\right)}\right|=\gamma_{r}\left|p_{k\left(\xi_{r}\right)}\right|
$$

implying that $p_{k\left(\xi_{r+1}\right)} \neq 0, \xi_{r+1} \neq 0$ and (iii) holds with $r+1$ in place of $r$. Also notice that, for $j \leq r-1$, we have $\xi_{r}, h_{r}, f_{r} \perp n_{j}$, which implies that $\xi_{r+1} \perp n_{j}$. Furthermore,

$$
\begin{gathered}
\left(h_{r}, n_{r}\right)=\left(h_{r}, n_{r}-\operatorname{Proj}_{\operatorname{Span}\left(n_{1}, \ldots, n_{r-1}\right)} n_{r}\right)=\left|h_{r}\right|^{2}, \quad\left(f_{r}, n_{r}\right)=1, \\
\left(\xi_{r+1}, n_{r}\right)=\left(\xi_{r}, n_{r}\right)-\left(f_{r}, n_{r}\right)\left(n_{r}, \xi_{r}\right)=0,
\end{gathered}
$$

so that $\xi_{r+1} \perp n_{j}$ for all $j \leq r$ and (ii) holds with $r+1$ in place of $r$. Properties (v) and (vi) hold with $r+1$ in place of $r$ by the assumption and construction.

Finally,

$$
\left|\xi_{r+1}-\xi_{r}\right|=\left|f_{r}\right| \cdot\left|\left(n_{r}, \xi_{r}\right)\right| \leq \kappa \varepsilon_{r} \delta\left|p_{k\left(\xi_{r}\right)}\right|=\gamma_{r} \delta\left|p_{k\left(\xi_{r}\right)}\right|,
$$

so that (iv) holds with $r+1$ in place of $r$.

Thus, if (4.3) is violated, we can find objects $\Gamma_{i}, \xi_{i} \neq 0$ having the properties (i)-(vi) with $r+1$ in place of $r$. This recursive process will stop at least when $r$ reaches $d+1$, just because property (i) will prevent us from finding $n_{r+1}$, which implies that at least at this moment (4.3) should be satisfied. This proves the theorem.

\section{Additional information}

Remark 5.1. One can estimate $p_{k}(x)$ from below for $x \in P^{o}$. It turns out that

$$
p_{k}(x) \geq \frac{d\left(x, x-a_{k}\right)}{n d\left(x, x-a_{k}\right)+n\left|x-a_{k}\right|},
$$

where $n d\left(x, x-a_{k}\right)+n\left|x-a_{k}\right|$ is obviously bounded away from zero. This and the fact that $\left|\nabla p_{k}(x)\right|$ is bounded, actually, show that $p_{k}(x)$ behaves like $d\left(x, x-a_{k}\right)$.

Indeed, take any $\xi \in \mathbb{R}^{d} \backslash\{0\}$ and observe that $y:=x+\xi d(x, \xi) /|\xi| \in P$ can be written as

$$
y=\sum_{k} q_{k} a_{k}, \quad q_{k} \geq 0, \quad \sum_{k} q_{k}=1
$$

Then

$$
\xi d(x, \xi) /|\xi|=y-x=\sum_{k}\left(p_{k}(x)-q_{k}\right)\left(x-a_{k}\right)
$$


and by (3.2)

$$
U_{(\xi)}(x) d(x, \xi) /|\xi|=\sum_{k} \frac{q_{k}-p_{k}(x)}{p_{k}(x)}=\sum_{k} \frac{q_{k}}{p_{k}(x)}-n \geq-n,
$$

so that, for any $\xi \neq 0$

$$
U_{(\xi)}(x) \geq-\frac{n|\xi|}{d(x, \xi)}
$$

If $\xi=x-a_{k}$, this and (3.3) imply that

$$
\frac{1}{p_{k}(x)}=n-U_{\left(x-a_{k}\right)}(x) \leq n+\frac{n\left|x-a_{k}\right|}{d\left(x, x-a_{k}\right)},
$$

which is equivalent to (5.1).

Remark 5.2. If $\xi=\sum_{k} q_{k}\left(a_{k}-x\right)$ and $\sum_{k} q_{k}=0$ then

$$
\sum_{k} \frac{q_{k}^{2}}{p_{k}^{2}(x)}=\sum_{k} \frac{\left(p_{k(\xi)}(x)\right)^{2}}{p_{k}^{2}(x)}+\sum_{k} \frac{\left(p_{k(\xi)}(x)-q_{k}\right)^{2}}{p_{k}^{2}(x)} .
$$

Indeed, write

$$
\sum_{k} \frac{q_{k}^{2}}{p_{k}^{2}(x)}=\sum_{k} \frac{\left(q_{k}-p_{k(\xi)}\right)^{2}}{p_{k}^{2}(x)}+2 \sum_{k} \frac{p_{k(\xi)}\left(q_{k}-p_{k(\xi)}\right)}{p_{k}^{2}(x)}+\sum_{k} \frac{\left(p_{k(\xi)}\right)^{2}}{p_{k}^{2}(x)}
$$

and observe that the middle term on the right is zero due to (3.5) and (3.6).

Remark 5.3. One can improve the estimate of $\left|p_{k\left(x-a_{k}\right)}\right|$ from Lemma 3.5. It turns out that

$$
1 \geq p_{k\left(x-a_{k}\right)}+1-p_{k}=\alpha_{k} p_{k}^{2}
$$

where

$$
\alpha_{k}=\frac{\left(p_{k\left(x-a_{k}\right)}+1-p_{k}\right)^{2}}{p_{k}^{2}}+\sum_{i \neq k} \frac{\left(p_{i\left(x-a_{k}\right)}-p_{i}\right)^{2}}{p_{i}^{2}} .
$$

In particular, $p_{k} \geq p_{k\left(x-a_{k}\right)} \geq p_{k}-1$.

Indeed, we may concentrate on proving (5.3) only for $k=1$ in which case we apply (5.2) with $\xi=x-a_{1}$. Since

$$
x-a_{1}=\left(p_{1}-1\right)\left(a_{1}-x\right)+p_{2}\left(a_{2}-x\right)+\ldots+p_{n}\left(a_{n}-x\right)
$$

one can take $q_{1}=p_{1}-1, q_{2}=p_{2}, \ldots, q_{n}=p_{n}$. Then by (3.5) and (5.2) (recall that $\left.a_{1}=0\right)$

$$
n-1+\frac{\left(p_{1}-1\right)^{2}}{p_{1}^{2}}=-U_{(x)(x)}+\alpha_{1} .
$$

On the other hand, by differentiating (3.3) we find

$$
\frac{p_{1(x)}}{p_{1}^{2}}=U_{(x)(x)}+U_{(x)}=U_{(x)(x)}+n-\frac{1}{p_{1}} .
$$

Hence,

$$
n-1+\frac{\left(p_{1}-1\right)^{2}}{p_{1}^{2}}=-\frac{p_{1(x)}}{p_{1}^{2}}+n-\frac{1}{p_{1}}+\alpha_{1},
$$




$$
-2 p_{1}+1=-p_{1(x)}-p_{1}+\alpha_{1} p_{1}^{2}
$$

and the equality in (5.3) follows. The first inequality follows from the fact that $\alpha_{1} p_{1}^{2} \geq\left(p_{1(x)}+1-p_{1}\right)^{2}$.

Remark 5.4. On can combine Remark 5.1 and the fact that for any $j, k$

$$
\left|p_{k\left(x-a_{j}\right)}(x)\right| \leq(n+1)^{1 / 2} p_{k}(x) / p_{j}(x) .
$$

in order to investigate the behavior of $\nabla p_{i}(x)$ as $x$ approaches $\partial P$. The above mentioned fact follows from (3.7) when $\xi=x-a_{j}$.

Remark 5.5. The functions $p_{k}$ have a peculiar symmetry. It turns out that for all $j, k$

$$
\frac{p_{k\left(x-a_{j}\right)}}{p_{k}^{2}}+\frac{1}{p_{j}}=\frac{p_{j\left(x-a_{k}\right)}}{p_{j}^{2}}+\frac{1}{p_{k}} .
$$

Indeed, differentiating (3.3) easily yields

$$
\frac{p_{k\left(x-a_{j}\right)}}{p_{k}^{2}}+\frac{1}{p_{j}}-n=U_{\left(x-a_{k}\right)\left(x-a_{j}\right)},
$$

where the right-hand side is symmetric with respect to $j, k$. One can combine (5.4) with Remarks 5.1 and 5.4 to obtain some information about the behavior of the second-order derivatives of $U$ near the boundary of $P$. For instance, $\left|U_{\left(x-a_{k}\right)\left(x-a_{j}\right)}\right| \leq n+2(n+1)^{1 / 2}\left(p_{k} p_{j}\right)^{-1}$.

Remark 5.6. Lemma 3.2 allows one to obtain a precise information about the behavior of the first-order derivatives of $U$ near the vertices of $P$. Indeed, (3.3) shows that

$$
\lim _{x \rightarrow a_{k}} U_{\left(x-a_{k}\right)}(x)=n-1 .
$$

Remark 5.7. If in the situation of Lemma 3.7 we have $\lambda(x) x \in P^{o}$ then the derivative with respect to $\lambda$ of the left-hand side of (3.8) is zero at $\lambda=\lambda(x)$. In this case, by substituting $\lambda=\lambda(x)$ into (3.8) and differentiating with respect to $x$ we find

$$
\lambda(x) U_{n-1, x^{i}}(\lambda(x) x)=U_{x^{i}}(x), \quad i=1, \ldots, d .
$$

In particular, the gradients of $U_{n-1}$ and $U$ are proportional at corresponding points.

\section{REFERENCES}

[1] G. Barles and E.R. Jakobsen, Error bounds for monotone approximation schemes for parabolic Hamilton-Jacobi-Bellman equations, Preprint.

[2] J.F. Bonnans, E. Ottenwaelter, and H. Zidani, A fast algorithm for the two dimensional HJB equation of stochastic control, M2AN Math. Model. Numer. Anal., Vol. 38 (2004), No. 4, 723-735.

[3] Hongjie Dong and N.V. Krylov, On the rate of convergence of finite-difference approximations for parabolic Bellman equations with Lipschitz coefficients in cylindrical domains, to appear in Applied Math. and Optimization.

[4] M.I. Freidlin, The factorization of nonnegative definite matrices, Teor. Verojatnost. i Primenen., Vol. 13 (1968), No. 2, 375-378 in Russian. 
[5] D. Gilbarg and N.S. Trudinger, "Elliptic partial differential equations of second order", 2nd edition, Grundlehren der mathematischen Wissenschaften, Vol. 224, Springer, Berlin-Heidelberg-New York-Tokyo, 1983.

[6] N.V. Krylov, "Nonlinear elliptic and parabolic equations of second order", Reidel, Dordrecht, 1987.

[7] N.V. Krylov, A priori estimates of smoothness of solutions to difference Bellman's equations with linear and quasilinear operators, Math. Comp., Vol. 76 (2007), 669698.

[8] H.J. Kushner and P.G. Dupuis, "Numerical methods for stochastic control problems in continuous time", 2nd edition, Springer Verlag, 2001.

[9] T. Motzkin and W. Wasow, On the approximation of linear elliptic differential equations by difference equations with positive coefficients, J. Math and Phys., Vol. 31 (1952), 253-259.

[10] R. S. Phillips and L. Sarason, Elliptic-parabolic equations of the second order, J. Math. Mech., Vol. 17 (1968), No. 2, 891-917.

127 Vincent Hall, University of Minnesota, Minneapolis, MN 55455, USA

E-mail address: krylov@math.umn.edu 